\title{
Bifurcation Analysis of a Rigid Impact Oscillator with Bilinear Damping
}

\author{
Liping Zhang $\left(\mathbb{D},{ }^{1}\right.$ Haibo Jiang $\left(\mathbb{D},{ }^{1}\right.$ and Yang Liu $\mathbb{D}^{2}$ \\ ${ }^{1}$ School of Mathematics and Statistics, Yancheng Teachers University, Yancheng 224002, China \\ ${ }^{2}$ College of Engineering, Mathematics and Physical Sciences, University of Exeter, Rennes Drive, Exeter, EX4 4RN, UK \\ Correspondence should be addressed to Haibo Jiang; yctcjhb@126.com
}

Received 24 September 2017; Revised 28 January 2018; Accepted 11 February 2018; Published 12 March 2018

Academic Editor: Hassan Haddadpour

Copyright (c) 2018 Liping Zhang et al. This is an open access article distributed under the Creative Commons Attribution License, which permits unrestricted use, distribution, and reproduction in any medium, provided the original work is properly cited.

\begin{abstract}
This paper studies a rigid impact oscillator with bilinear damping developed as the mechanical model of an impulsive switched system. The stability and the bifurcation of periodic orbits in the impact oscillator are determined by using the mapping methods. One-parameter bifurcation analyses under variation of forcing frequency and amplitude of external excitation are carried out. Coexisting attractors and various types of bifurcations, such as grazing, period-doubling, and saddle-node, are observed, which show the complex phenomena inhered in this impact oscillator.
\end{abstract}

\section{Introduction}

The rigid impact, known as the impulsive reactions whenever rigid bodies collide, widely exists in many engineering applications, such as rotating machinery, car suspension systems, and cutting processes. In general, such impact can be studied using the rigid impact oscillator, which is modelled using the coefficient of restitution rule assuming the instantaneous reversal of velocity for the collision body. The rigid impact oscillator is a nonsmooth dynamical system which can exhibit complex dynamical behavior, so the stability and the bifurcation of the rigid impact oscillator have received great attention; see, for example, [1-18]. In [1], Shaw and Holmes observed the chaotic and long period motions in a class of periodically forced linear oscillators with impacts. In [2], Whiston analyzed the vibroimpact response of a onedimensional undamped linear oscillator preloaded against a stop by a constant force and subjected to harmonic excitation. Nordmark [3] studied the singularities of grazing impacts in a single-degree-of-freedom periodically forced oscillator subjected to a rigid amplitude constraint using analytical methods. In [4], Ivanov developed the linear theory for analyzing the stability and the bifurcation of a class of rigid impact oscillators. Foale $[5,6]$ classified the types of grazing bifurcation in a class of rigid impact oscillators and presented analytical results to show how the type of grazing bifurcation changed with control parameter. In [7], Luo and Xie observed Hopf bifurcations in a vibroimpact system at two strong resonance cases. Luo and Chen [8] investigated the mapping dynamics of periodic motions for a class of rigid impact oscillators. Dankowicz and Zhao $[9,10]$ studied different bifurcation scenarios associated with the switching between impacting and nonimpacting motions of impact actuators near grazing. In [11], Thota and Dankowicz studied continuous and discontinuous grazing bifurcations of impacting oscillators by using the discontinuity mapping. In particular, the concept of discontinuity geometry was introduced in [1214] to study the grazing bifurcation in rigid impact oscillators. Later on, Mason and Piiroinen [15] focused on the intricate relationship between smooth and nonsmooth phenomena in an impacting system. Jiang et al. [16] studied the antiphase synchronization and the symmetry-breaking bifurcation of two mechanical oscillators coupled by rigid impacts. In [17], Jiang et al. investigated the differences between the grazinginduced bifurcations in impact oscillators with one-sided elastic and rigid constraints by a path-following method.

In this paper, we will study a rigid impact oscillator with bilinear damping through one-parameter bifurcation analysis. In general, linear damping is always considered in the physical models of mechanical systems. However, in some 


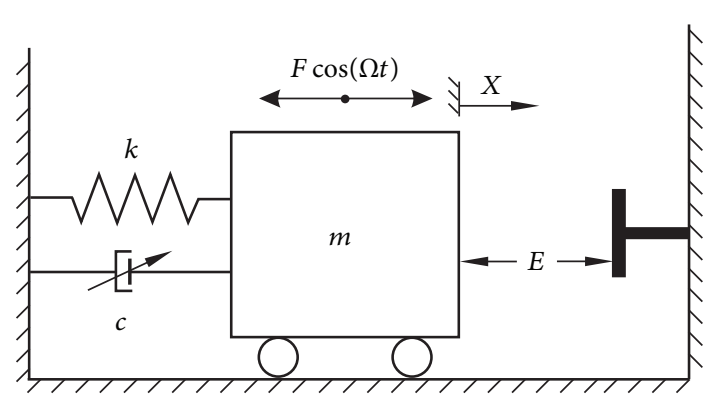

(a)

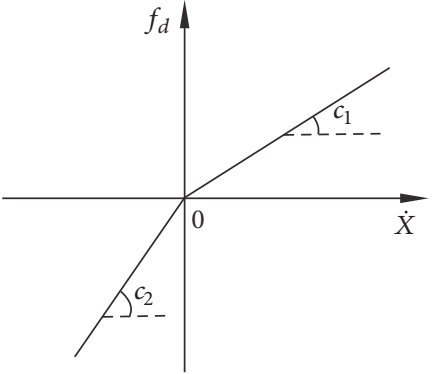

(b)

Figure 1: (a) Physical model and (b) the dual-rate damper model.

engineering practices, piecewise linear or viscous dampers are widely designed and used owing to their adaptability in the changing environment. For example, shock absorber with bilinear characteristics is often used for improving the driving safety and traveling comfort of vehicles [19]. Therefor, piecewise linear or viscous dampers have received considerable attention from researchers and engineers, for example, [2029]. In [19], the nonlinear stationary oscillations of a quartervehicle model with two degrees of freedom subjected to a vertical road excitation and bilinear damping were studied. In [20], Natsiavas presented an appropriate stability analysis for periodic solutions of harmonically excited piecewise linear systems with dry friction and damping coefficients depending on the velocity direction. Natsiavas and Verros [21] studied the dynamics of oscillators with strongly nonlinear asymmetric damping. In [22], Verros et al. studied the dynamic response of a controlled single-degree-of-freedom quarter-car model subjected to road excitation, and the control strategy applied to the system was based on the selection of two values of the damping ratio. In [23], the effect of asymmetric damping on the sprung mass position was investigated by analyzing a two-degree-of-freedom quartervehicle model. In [24], Verros et al. investigated the car models involving passive damping with constant and dualrate characteristics. In [25], the model of a single degree of system subjected to harmonic base excitation was used to study the performance of vibration isolation using four semiactive dampers, and five control algorithms were studied based on the skyhook control, the balance control, and the adaptive damping control. Papalukopoulos and Natsiavas [26] investigated biodynamic response of the lumped parameter passenger-seat models coupled with the simplified models of ground vehicles, and dual-rate suspension damping was used to model the damper between the vehicles. In [27], Luo and Rajendran investigated the periodic motions and the stability in a semiactive suspension system with the magnetorheological damping which varies with relative velocity. In addition, Waters et al. [28] considered the effect of dual-rate suspension damping on vehicle response to transient road inputs. A damping control law was proposed in [29] to adjust the damping coefficient for suppressing the chaotic behavior of an impact oscillator.

Recently, there has been an increasing interest in the analysis and synthesis of impulsive switched systems with the presence of two nonsmooth effects, impulsive disturbances and switching, because of their significance in theory and applications in physics, biology, engineering, and information science [30-37]. In [31], Xie and Wang introduced the impulsive behavior into switched linear systems and studied their controllability and observability. Guan et al. [32] studied the exponential and asymptotical stability for a class of hybrid impulsive and switching nonlinear systems and developed a new hybrid impulsive and switching control strategy. In [33], Zhong and Wu studied the robust exponential stability for a class of switched systems with impulsive effect and structural perturbations. In [34], $H_{\infty}$ control of a class of discrete impulsive switched systems with disturbance was studied by using the Lyapunov direct method. In [35-37], the stability analysis of impulsive switched systems with time delays was studied. In this paper, we will develop a mechanical model for impulsive switched systems and carry out bifurcation analysis for this system [38]. Firstly, the physical model of a rigid impact oscillator with bilinear damping is built, and the dualrate damper model is used to describe the bilinear damping. Secondly, in order to describe the segments of periodic orbits of the impact oscillator, we divide the phase space into four subspaces and obtain four discontinuity boundaries. Then, five local maps are defined between these discontinuity boundaries, and periodic orbits can be described by the composite of these local maps. Thus, the stability and the bifurcation of the impact oscillator can be determined by the eigenvalues of the Jacobian matrix of these global maps. Thirdly, one-parameter bifurcation analysis is carried out to show the influence of forcing frequency and amplitude on the dynamics of the system. Finally, numerical simulations show that the system exhibits complex phenomena, including periodic and chaotic orbits.

\section{Physical Model}

The physical model of the rigid impact oscillator with onesided constraint is shown in Figure 1(a). Displacement of the mass is represented by $X$. The mass is connected to the supporting base by a linear spring with stiffness $k$ and a dualrate damper with the damping coefficient expressed as

$$
c= \begin{cases}c_{1}, & \dot{X} \geq 0, \\ c_{2}, & \dot{X}<0,\end{cases}
$$




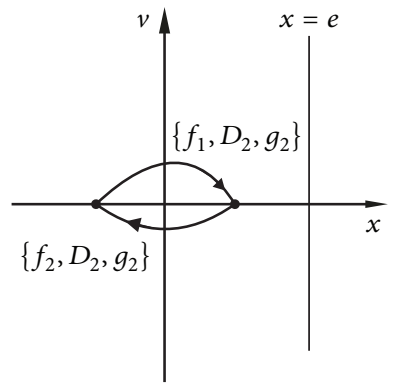

(a)

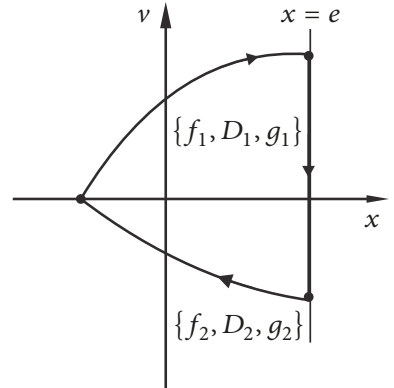

(b)

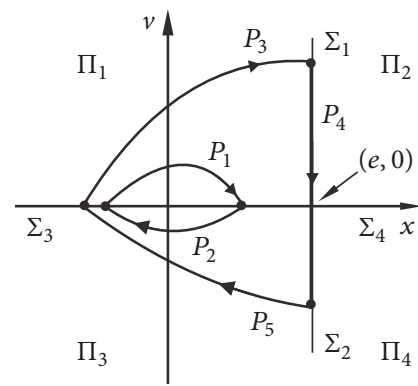

(c)

FIGURE 2: Representative trajectories and segments of (a) the nonimpacting period-1 orbit, (b) the period-1 orbit with one impact per period, and (c) the local maps.

where $\dot{X}$ denotes the differentiation of $X$ with respect to the time $t$. The relationship between the damping force $f_{d}=$ $c \dot{X}$ and the velocity $V=\dot{X}$ for the dual-rate damper model is presented in Figure 1(b). As shown in the figure, the damping coefficients in compression and extension are different depending on the sign of the velocity. Thus the dualrate damper is easy to be designed in engineering and it can also achieve a better compromise performance for the vehicle between ride, road-holding, handling, and control, so it is used as a typical model in automotive engineering applications. However, to adapt complex engineering circumstances, we can consider the other types of dampers, such as three-linear, four-linear, and viscous. The dynamics of impact oscillators with these dampers may be complex, which needs further studies. External harmonic excitation is applied on the mass with amplitude $A$ and frequency $\Omega$. The gap between the mass and the stop is denoted by $E$, and the stop is assumed to be rigid and described by the coefficient of restitution $r$.

The governing equations of the impact oscillator can be written as

$$
\begin{aligned}
m \ddot{X}+c \dot{X}+k X & =F \cos (\Omega t), \quad X<E, \\
\dot{X}^{+} & =-r \dot{X}^{-}, \quad X=E,
\end{aligned}
$$

where $\dot{X}^{-}$and $\dot{X}^{+}$represent the velocities of the impact oscillator before and after impact, respectively. For simplicity, we introduce the following nondimensional variables and parameters, $\tau=\Omega_{0} t, \Omega_{0}=\sqrt{k / m}, x=X / y_{0}, e=E / y_{0}$, $\xi=c /\left(2 m \Omega_{0}\right), \xi_{1}=c_{1} /\left(2 m \Omega_{0}\right), \xi_{2}=c_{2} /\left(2 m \Omega_{0}\right)$, and $A=F /\left(k y_{0}\right)$, where $\tau$ is the nondimensional time, $x$ is the nondimensionalized displacement, $e$ is the nondimensional gap, $A$ is the nondimensional forcing amplitude, and $y_{0}$ is an arbitrary reference distance. After the nondimensionalization, the equations of motion of the impact oscillator taking into account the dual-rate damper are given as

$$
\begin{array}{r}
x^{\prime \prime}+2 H\left(x^{\prime}\right) \xi_{1} x^{\prime}+2 H\left(-x^{\prime}\right) \xi_{2} x^{\prime}+x=A \cos (\omega \tau), \\
x<e, \\
\left(x^{\prime}\right)^{+}=-r\left(x^{\prime}\right)^{-}, \\
x=e,
\end{array}
$$

where $x^{\prime}$ denotes the differentiation of $x$ with respect to the nondimensional time $\tau$ and $H(\cdot)$ is the Heaviside step function.

\section{Local Maps and Stability Analysis}

The impact oscillator (3) is a state-dependent impulsive switched (hybrid) dynamical system which can be expressed as

$$
\begin{aligned}
& x^{\prime \prime}(\tau)=f_{p}\left(\tau, x(\tau), x^{\prime}(\tau)\right), \quad\left(x(\tau), x^{\prime}(\tau)\right) \in C_{p}, \\
& x^{\prime}\left(\tau^{+}\right)=g_{q}\left(x^{\prime}\left(\tau^{-}\right)\right), \quad\left(x(\tau), x^{\prime}(\tau)\right) \in D_{q},
\end{aligned}
$$

where $p \in P=\{1,2\}, q \in Q=\{1,2\}, f_{1}\left(\tau, x(\tau), x^{\prime}(\tau)\right)=$ $A \cos (\omega \tau)-2 \xi_{1} x^{\prime}-x, f_{2}\left(\tau, x(\tau), x^{\prime}(\tau)\right)=A \cos (\omega \tau)-2 \xi_{2} x^{\prime}-$ $x, g_{1}\left(x^{\prime}\left(\tau^{-}\right)\right)=-r x^{\prime}\left(\tau^{-}\right), g_{2}\left(x^{\prime}\left(\tau^{-}\right)\right)=x^{\prime}\left(\tau^{-}\right), v=x^{\prime}, C_{1}=$ $\{(x, v) \mid x<e, v \geq 0\}, C_{2}=\{(x, v) \mid x<e, v<0\}, D_{1}=$ $\{(x, v) \mid x=e, v>0\}, D_{2}=\{(x, v) \mid x \leq e, v=0\}$.

Given an initial value $\left(x(\tau), x^{\prime}(\tau)\right) \in C_{p^{\prime}}, p^{\prime} \in P$, the motion of the impact oscillator is governed by the equation $x^{\prime \prime}(\tau)=f_{p^{\prime}}\left(\tau, x(\tau), x^{\prime}(\tau)\right)$ until $\left(x(\tau), x^{\prime}(\tau)\right) \in D_{q^{\prime}}, q^{\prime} \epsilon$ $Q$, and the state jumps according to $x^{\prime}\left(\tau^{+}\right)=g_{q^{\prime}}\left(x^{\prime}\left(\tau^{-}\right)\right)$. If $\left(x\left(\tau^{+}\right), x^{\prime}\left(\tau^{+}\right)\right) \in C_{p^{\prime \prime}}, p^{\prime \prime} \in P$, the motion switches to continuous motion governed by the equation $x^{\prime \prime}(\tau)=$ $f_{p^{\prime \prime}}\left(\tau, x(\tau), x^{\prime}(\tau)\right)$, and then, the procedure continues. Each segment can be denoted by the signature $\left\{f_{p}, D_{q}, g_{q}\right\}$. Figure 2(a) shows the representative trajectory of nonimpacting period-1 orbit by using the segments $\left\{f_{1}, D_{2}, g_{2}\right\}$ and $\left\{f_{2}, D_{2}, g_{2}\right\}$. Figure 2(b) presents the representative trajectory of period-1 orbit with one impact per period using the segments $\left\{f_{1}, D_{1}, g_{1}\right\}$ and $\left\{f_{2}, D_{2}, g_{2}\right\}$.

In the following, the mapping methods proposed in [8, $17,27,39-41]$ will be used to study the stability and the bifurcation of the impact oscillator (3). In order to describe the segments of the impact oscillator, we divide the phase space into four subspaces $\Pi_{1}, \Pi_{2}, \Pi_{3}$, and $\Pi_{4}$, where $\Pi_{1}=$ $\{(x, v ; \tau) \mid x<e, v>0\}, \Pi_{2}=\{(x, v ; \tau) \mid x>e, v>0\}$, $\Pi_{3}=\{(x, v ; \tau) \mid x<e, v<0\}$, and $\Pi_{4}=\{(x, v ; \tau) \mid$ $x>e, v<0\}$. Switches between these regimes occur on the discontinuity boundaries, $\Sigma_{1}, \Sigma_{2}, \Sigma_{3}$, and $\Sigma_{4}$, where $\Sigma_{1}=$ $\{(x, v ; \tau) \mid x=e, v>0\}, \Sigma_{2}=\{(x, v ; \tau) \mid x=e, v<0\}$, $\Sigma_{3}=\{(x, v ; \tau) \mid x<e, v=0\}$, and $\Sigma_{4}=\{(x, v ; \tau) \mid$ 
$x>e, v=0\}$. Here, we define five local maps as follows: $P_{1}: \Sigma_{3} \rightarrow \Sigma_{3}, P_{2}: \Sigma_{3} \rightarrow \Sigma_{3}, P_{3}: \Sigma_{3} \rightarrow \Sigma_{1}, P_{4}: \Sigma_{1} \rightarrow \Sigma_{2}$, and $P_{5}: \Sigma_{2} \rightarrow \Sigma_{3}$, which are shown in Figure 2(c). These local maps project the point located on one of the discontinuity boundaries onto another. The local map $P_{1}$ can be represented by $P_{1}:\left(x_{n}, v_{n} ; \tau_{n}\right) \rightarrow\left(x_{n+1}, v_{n+1} ; \tau_{n+1}\right)$, where $v_{n}=v_{n+1}=0$ and the trajectory is in the regime $\Pi_{1}$. The local map $P_{2}$ can be represented by $P_{2}:\left(x_{n}, v_{n} ; \tau_{n}\right) \rightarrow\left(x_{n+1}, v_{n+1} ; \tau_{n+1}\right)$, where $v_{n}=$ $v_{n+1}=0$ and the trajectory is in the regime $\Pi_{3}$. The local map $P_{3}$ can be represented by $P_{3}:\left(x_{n}, v_{n} ; \tau_{n}\right) \rightarrow\left(x_{n+1}, v_{n+1} ; \tau_{n+1}\right)$, where $v_{n}=0, x_{n+1}=e$, and the trajectory is in the regime $\Pi_{1}$. Due to impacts at the boundary, $P_{4}$ represents the impact relation; that is, $P_{4}:\left(x_{n}, v_{n} ; \tau_{n}\right) \rightarrow\left(x_{n+1}, v_{n+1} ; \tau_{n+1}\right)$, where $\tau_{n+1}=\tau_{n}, x_{n}=x_{n+1}=e, v_{n+1}=-r * v_{n}$. The local map $P_{5}$ can be represented by $P_{5}:\left(x_{n}, v_{n} ; \tau_{n}\right) \rightarrow\left(x_{n+1}, v_{n+1} ; \tau_{n+1}\right)$, where $x_{n}=e, v_{n+1}=0$, and the trajectory is in the regime $\Pi_{3}$. The governing equations for the local maps $P_{1}, P_{2}, P_{3}$, and $P_{5}$ can be obtained from the solutions of the linear ordinary differential equations in the corresponding regimes (see the Appendix). For example, the global map of the nonimpacting period-1 orbit shown in Figure 2(a) can be expressed as $P_{21}=$ $P_{2} \circ P_{1}$. Thus, the stability of this period-1 motion can be determined using the corresponding Jacobian matrix of the global map which can be computed by the chain rule $D P_{21}=$ $D P_{2} \times D P_{1}$. The global map of the period-1 orbit with one impact per period shown in Figure 2(b) can be expressed as $P_{543}=P_{5} \circ P_{4} \circ P_{3}$, and its Jacobian matrix can be computed by $D P_{543}=D P_{5} \times D P_{4} \times D P_{3}$. Therefore, for a periodic motion of mapping structure $P_{n_{k} \cdots n_{2} n_{1}} \equiv P_{n_{k}} \circ \cdots \circ P_{n_{2}} \circ P_{n_{1}}$, where $n_{i} \in\{1,2,3,4,5\}, i=1,2, \ldots, k$, its stability and bifurcations can be determined by the eigenvalues of the Jacobian matrix $D P_{n_{k} \cdots n_{2} n_{1}}=D P_{n_{k}} \times \cdots \times D P_{n_{2}} \times D P_{n_{1}}$. If two eigenvalues of the Jacobian matrix lie inside the unit circle, the periodic motion is stable. If one of them lies outside the unit circle, the periodic motion is unstable. If one of the two eigenvalues is +1 and the second one is inside the unit circle, the saddle-node bifurcation will occur. If one of the two eigenvalues is -1 and the second one is inside the unit circle, the period-doubling bifurcation may occur. In our numerical simulations, we will follow these bifurcations by using the numerical continuation method developed in $[40,41]$.

\section{Bifurcation Analysis of the Impact Oscillator with Bilinear Damping}

In this section, one-parameter bifurcation analysis of the impact oscillator with bilinear damping is carried out to investigate the influence of forcing frequency and amplitude on the dynamics of the system.

4.1. Influence of the Forcing Frequency. To investigate the influence of forcing frequency on the dynamics, we chose $a=0.3, e=1, \xi_{1}=0.05, \xi_{2}=0.04, r=0.7$, and the forcing frequency $\omega$ was considered as a branching parameter. Bifurcation diagrams were constructed by starting from an initial frequency, plotting the solution once it converged to a periodic response (or plotting the next 500 iterates if there was no convergence after 2000 periods), and using the final conditions as the initial conditions of the next value of frequency. Bifurcation diagram constructed by the mass displacement under variation of forcing frequency $\omega$ is shown in Figure 3. As can be seen from Figure 3, an interesting bifurcation structure was recorded. As the forcing frequency $\omega$ exceeds 0.8 , the nonimpacting period-1 orbit $\left(P_{21}\right)$ is unchanged until the orbit grazes the discontinuity boundary; that is, grazing bifurcation occurs at $\omega=\omega_{g 1} \approx 0.84382$. Then the period-1 response changes to a stable period-3 orbit with one impact $\left(P_{(21)^{2} 543}=P_{2121543}\right)$ via a grazing bifurcation. As the forcing frequency is further increased, the trajectory of the period-3 orbit with one impact per period contacts the discontinuity boundary with zero velocity again; that is, the second grazing bifurcation is observed at $\omega=$ $\omega_{s g} \approx 0.98108$ which leads to a small regime of chaotic motions for the impact oscillator. Thereafter, a reverse perioddoubling cascade occurs and the response of the impact oscillator changes from chaotic motion to a stable period-4 orbit with two impacts per period $\left(P_{(212543)^{2}}=P_{2154321543}\right)$ followed by a stable period- 2 orbit with one impact per period $\left(P_{212543}\right)$, which bifurcates into a stable period-1 orbit with one impact per period $\left(P_{543}\right)$ eventually. With further increase of the forcing frequency, the period-1 orbit with one impact undergoes a saddle-node bifurcation at $\omega=\omega_{\text {sn }} \approx 1.3846$ resulting in an unstable period-1 orbit with one impact per period $\left(P_{543}(U)\right)$. As the forcing frequency $\omega$ becomes less than 1.4 , there is a nonimpacting period-1 orbit $\left(P_{21}\right)$ followed by a grazing bifurcation occurring at $\omega=\omega_{g 2} \approx 1.1311$, and the state of the impact oscillator jumps to the coexisting stable period-1 orbit with one impact per period.

Figure 3(b) shows the corresponding amplitude frequency curve of the period-1 solution. As the frequency increases, a grazing bifurcation $\left(\mathrm{GR}_{1}\right)$ occurs at the critical value $\omega=\omega_{g 1}$, leading a stable nonimpacting period1 orbit to an unstable period-1 orbit with one impact per period. With the further increase in the frequency, a perioddoubling bifurcation (PD) takes place and the unstable period-1 orbit with one impact per period regains stability. Later on, the stable period-1 impact orbit with one impact per period loses stability via a saddle-node bifurcation (SN). On the other hand, as the frequency decreases, the second grazing bifurcation (GR) occurs at the critical value $\omega=$ $\omega_{g 2}$. Hence, the stable nonimpacting period-1 orbit becomes unstable, and an unstable period-1 orbit with one impact per period emerges. Then, the lower branch of unstable solutions coalesces with the upper stable branch and they annihilate each other through a saddle-node bifurcation (SN). Here, the hysteresis phenomenon is observed.

Figure 4 presents the evolution of basins of attraction of the impact oscillator with bilinear damping. Here, the basin of the period-1 orbit with one impact per period (red) is given in cyan, and the nonimpacting period-1 orbit (blue) is shown in magenta. As can be seen from this series of graphs, the basin for the period-1 orbit with one impact per period gradually shrinks as the frequency increases and disappears completely at $\omega=\omega_{s n} \approx 1.3846$, while the nonimpacting period-1 orbit gradually grows as the frequency increases.

4.2. Influence of the Forcing Amplitude. To investigate the influence of forcing amplitude on the dynamics, we fixed 

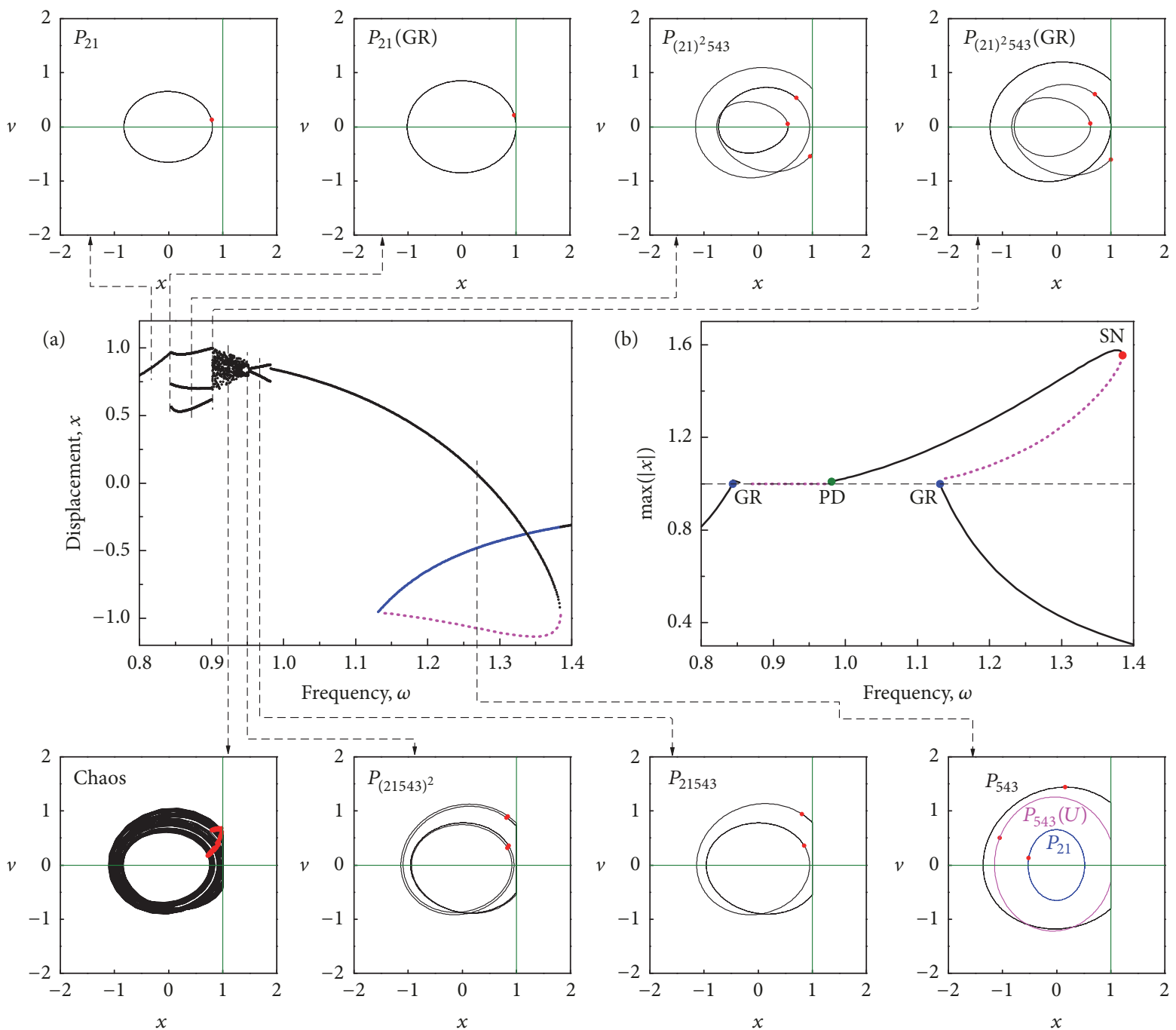

Figure 3: (a) Bifurcation diagrams constructed for the mass displacement under varying forcing frequency $\omega$ calculated at $a=0.3, e=$ $1, \xi_{1}=0.05, \xi_{2}=0.04, r=0.7$. Black and blue dots mark the mass displacement which is swept in the increasing and decreasing directions, respectively. The magenta dots show the unstable period-1 orbit with one impact. Additional windows demonstrate the trajectories of the attractors on the phase plane $(x, v)$ obtained for $\omega=0.83, \omega=\omega_{g 1} \approx 0.84382, \omega=0.87, \omega=0.90108, \omega=0.91, \omega=0.95135, \omega=0.96$, and $\omega=1.25$ (stable impacting period-1 orbit in black, unstable impacting period-1 orbit in magenta, stable nonimpacting period-1 orbit in blue). Green solid lines indicate the location of the discontinuity boundaries $x=e$ and $v=0$, and the red points show the Poincaré sections. (b) The corresponding amplitude frequency curve showing the maximum of the absolute values of the mass displacement as a function of the forcing frequency $\omega$. Blue, green, and red dots represent one-parameter grazing (GR), period-doubling (PD), and saddle-node (SN) bifurcations, respectively. Solid and dotted lines denote stable and unstable solutions, respectively. The dashed horizontal line denotes $\max (\operatorname{abs}(x))=1$.

$\omega=0.8, e=1, \xi_{1}=0.05, \xi_{2}=0.04, r=0.7$, and used the forcing amplitude $a$ as a branching parameter. The constructed bifurcation diagram is shown in Figure 5. As can be seen from the figure, an interesting bifurcation structure was recorded. As the forcing amplitude $a$ exceeds 0.3 , the nonimpacting period-1 orbit $\left(P_{21}\right)$ is unchanged until the orbit grazes the discontinuity boundary $x=e$, so the grazing bifurcation (GR) occurs at $a=a_{g} \approx 0.36981$. Then, a small regime of chaotic motions can be observed which is followed by a period- 2 orbit with one impact per period $\left(P_{21543}\right)$. As the forcing amplitude increases, the trajectory of the period2 orbit with one impact contacts the discontinuity boundary $x=e$ with zero velocity, so the second grazing bifurcation
(GR) occurs at $a=a_{\text {sg }} \approx 0.44681$ leading to a period-2 orbit with two impacts per period $\left(P_{(212543)^{2}}=P_{2154321543}\right)$. Shortly after, a reverse period-doubling bifurcation (PD) occurs and the response of the impact oscillator changes to a stable period-1 orbit with one impact per period $\left(P_{21543}\right)$. With further increase in the forcing amplitude, a saddle-node bifurcation (SN) is observed at $a=a_{\mathrm{sn} 1} \approx 2.0729$, and the state changes to a coexisting stable period-1 orbit with two impacts per period $\left(P_{(543)^{2}}=P_{543543}\right)$. Now we consider the variation of the branching parameter in a reversed direction. As the forcing amplitude $a$ becomes less than 2.4, there is a stable period-1 orbit with two impacts per period $\left(P_{(543)^{2}}=\right.$ $\left.P_{543543}\right)$ which is followed by a saddle-node bifurcation (SN) 


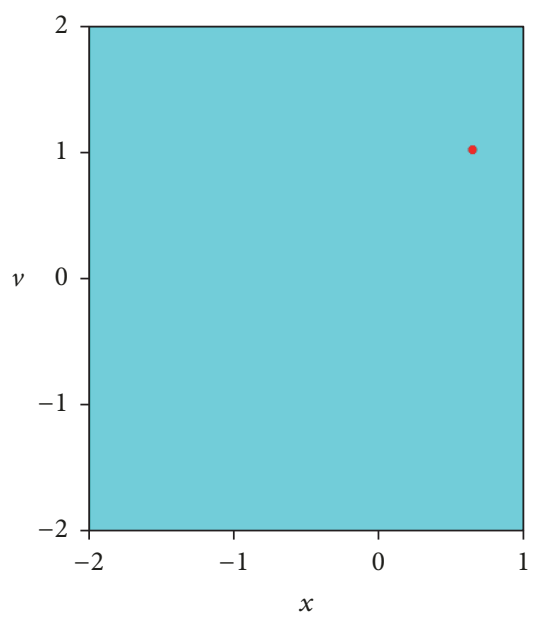

(a)

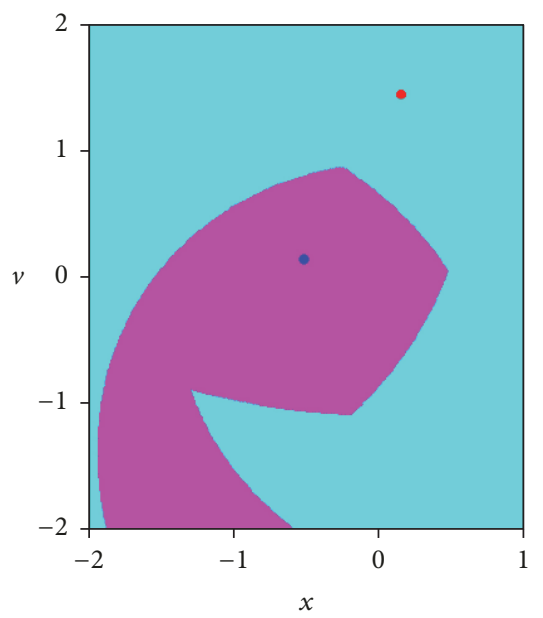

(d)

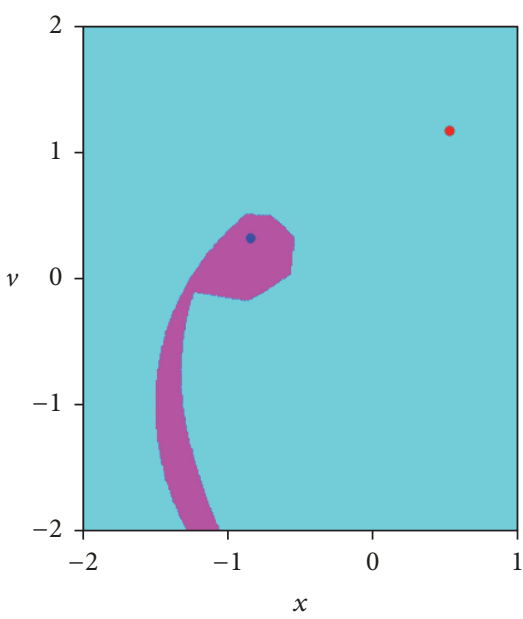

(b)

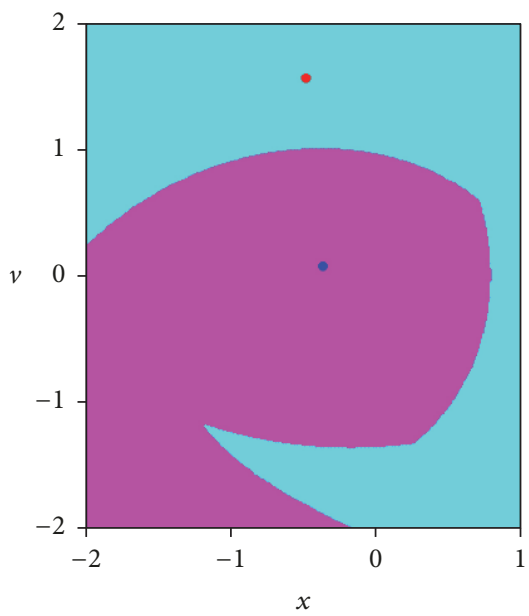

(e)

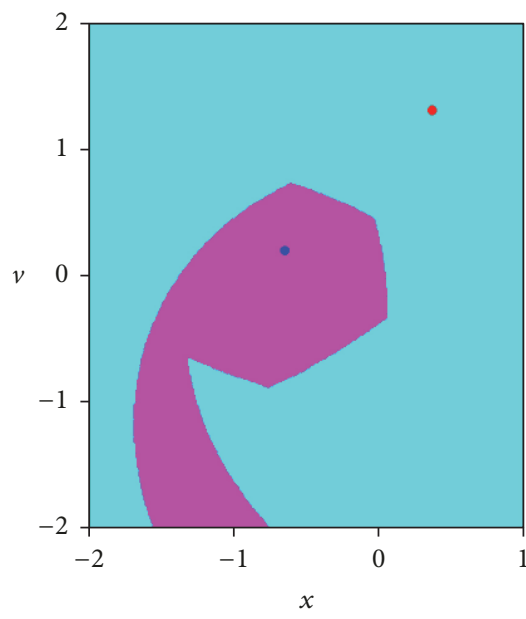

(c)

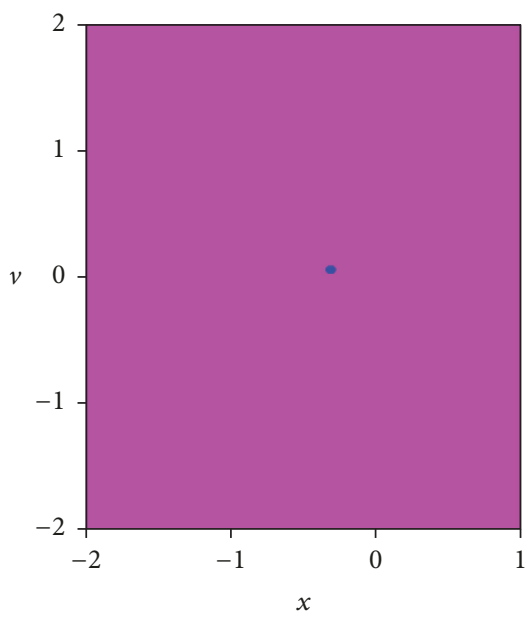

(f)

FIGURE 4: Evolution of the basins of attraction for the impact oscillator with bilinear damping obtained for $a=0.3, e=1, \xi_{1}=0.05, \xi_{2}=$ $0.04, r=0.7$, (a) $\omega=1.10$, (b) $\omega=1.15$, (c) $\omega=1.20$, (d) $\omega=1.25$, (e) $\omega=1.35$, and (f) $\omega=1.40$. The period-1 orbit with one impact per period is shown in red with a cyan basin, and the nonimpacting period-1 orbit is presented in blue with a magenta basin.

recorded at $a=a_{\mathrm{sn} 2} \approx 1.6081$. Since then, the state of the impact oscillator jumps to the coexisting stable period-1 orbit with one impact per period $\left(P_{21543}\right)$. Again, the hysteresis phenomenon is observed.

Figure 6 presents the evolution of basins of attraction for the impact oscillator with bilinear damping. Here the basin of the period-1 orbit with one impact per period (red) is given in cyan and the period-1 orbit with two impacts per period (blue) is in magenta. As can be seen from these graphs, the basin of the period-1 orbit with one impact per period shrinks gradually as the frequency increases and disappears completely at $a=a_{\mathrm{sn} 1} \approx 2.0729$. The basin for the period1 orbit with two impacts per period grows gradually as the amplitude increases.

\section{Conclusions}

The rigid impact oscillator with bilinear damping constructed as the mechanical model of an impulsive switched system was investigated through one-parameter bifurcation analysis in this paper. The stability and the bifurcation of the periodic orbits in the impact oscillator were studied by using the mapping methods. In order to describe the periodic orbit, we divided the phase space of the system into four subspaces and defined four discontinuous boundaries. Local maps were built to describe the motion of the system, and the eigenvalues of the Jacobian matrix of these global maps were used to determine the stability and bifurcation of the system.

Our one-parameter bifurcation analyses show that, as the forcing frequency varies for $\omega \in[0.8,1.4]$, the system experiences grazing, period-doubling, and saddle-node bifurcations. The coexistence of impacting period- 1 and nonimpacting period-1 orbits was observed for $\omega \in[0.98108,1.3846]$. A series of basins of attraction of the impact oscillator were presented to show the evolution of these two coexisting attractors, where the basin for the impacting period-1 orbit gradually shrinks as the forcing frequency increases and disappears completely at $\omega \approx 1.3846$. 


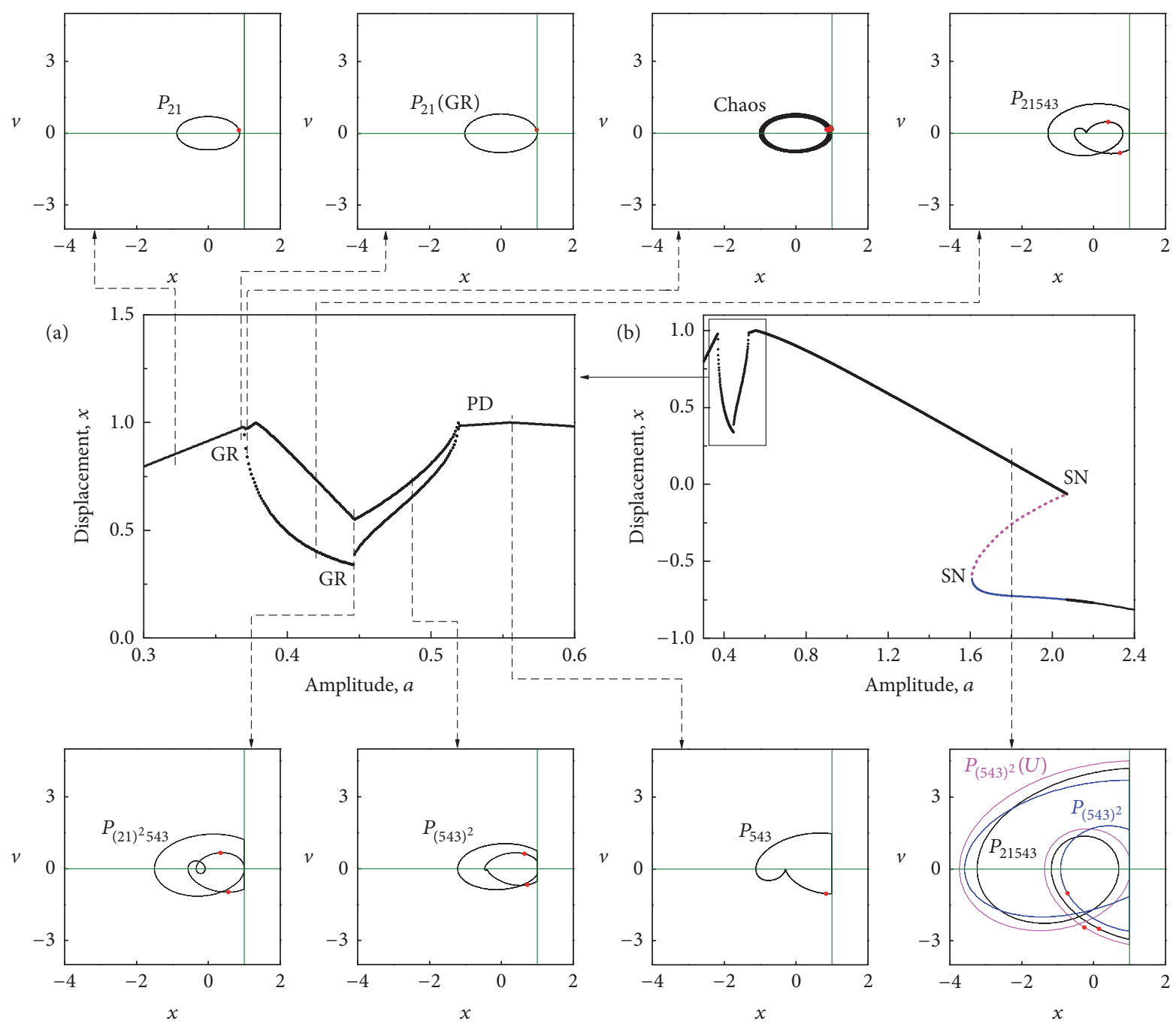

FIGURE 5: Bifurcation diagrams constructed for the mass displacement under varying forcing amplitude (a) $a \in[0.3,0.6]$ and (b) $a \in[0.6,2.4]$ calculated at $\omega=0.8, e=1, \xi_{1}=0.05, \xi_{2}=0.04$, and $r=0.7$. Black and blue dots show the mass displacements swept in increasing and decreasing directions, respectively. The magenta dots show the unstable period-1 orbit with one impact. Additional windows demonstrate the trajectories of the attractors on the phase plane $(x, v)$ obtained for $a=0.32, a=0.36981, a=0.36991, a=0.42007, a=0.44681, a=$ $0.48486, a=0.55328$, and $a=1.8$, respectively. Stable period-1 orbit with two impacts per period, unstable period- 1 orbit with two impacts per period, and stable period-1 orbit with one impact per period are shown in blue, magenta, and black. Green lines indicate the location of the discontinuity boundaries $x=e$ and $v=0$, and the red points show the Poincaré sections.

Our one-parameter bifurcation analyses using the forcing amplitude as the branching parameter reveal that the impact oscillator encounters two grazing, one period-doubling, and two saddle-node bifurcations when the forcing amplitude varies for $a \in[0.3,2.4]$. When $a \in[1.6081,2.0729]$, a period-1 orbit with one impact per period and a period-1 orbit with two impacts per period coexist. The basins of attraction show that the period-1 orbit with one impact disappears completely at $a \approx 2.0729$, and the basin for the period-1 orbit with two impacts grows gradually as the forcing amplitude increases.

Compared to the research on the rigid impact oscillators with linear damping in [1-18], the bilinear damping was considered and the mapping method was adopted to investigate the dynamics of the rigid impact oscillator with bilinear damping focusing on grazing bifurcations, which can be utilized for analyzing this type of nonsmooth dynamical systems. Compared to the research on piecewise linear dampers in [19-29], numerical methods including bifurcation diagrams, phase-portraits, and basin of attraction were carried out to show the influence of the forcing frequency and amplitude on the dynamics of the system. Furthermore, coexisting attractors and various types of bifurcations, such as grazing, period-doubling, and saddle-node, are observed, which show the complex phenomena inhered in the impact oscillator. Future works include two-parameter bifurcation analysis and investigation of the bifurcation mechanism inhered in this impact oscillator. 


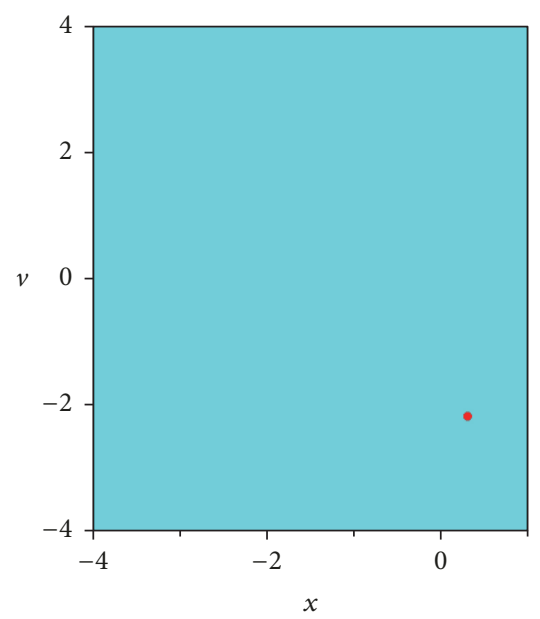

(a)

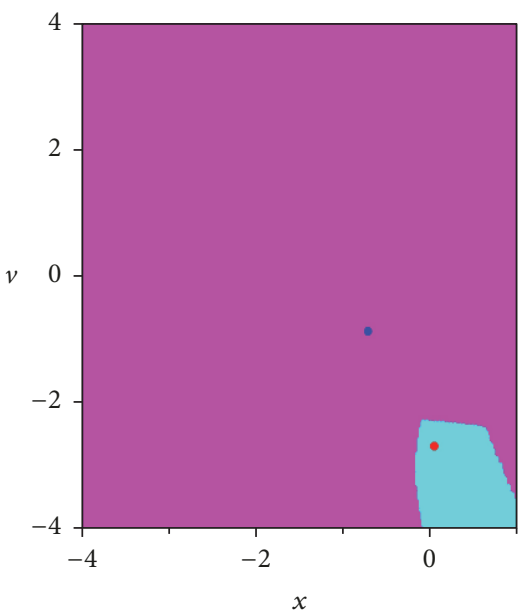

(d)

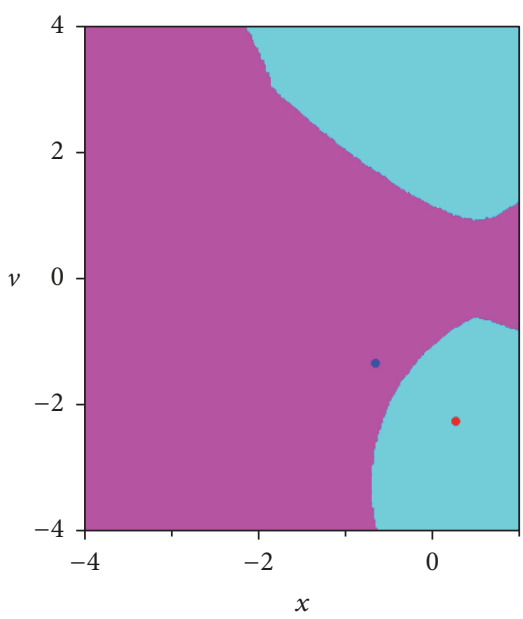

(b)

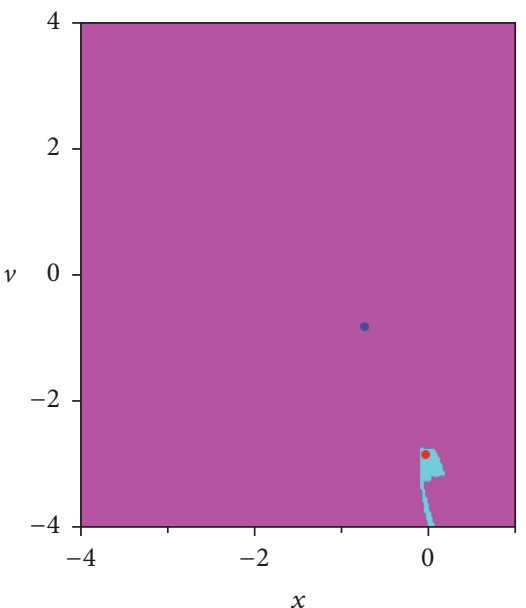

(e)

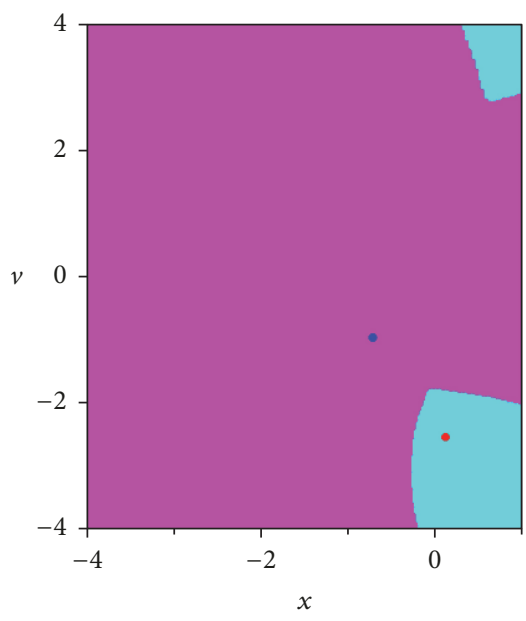

(c)

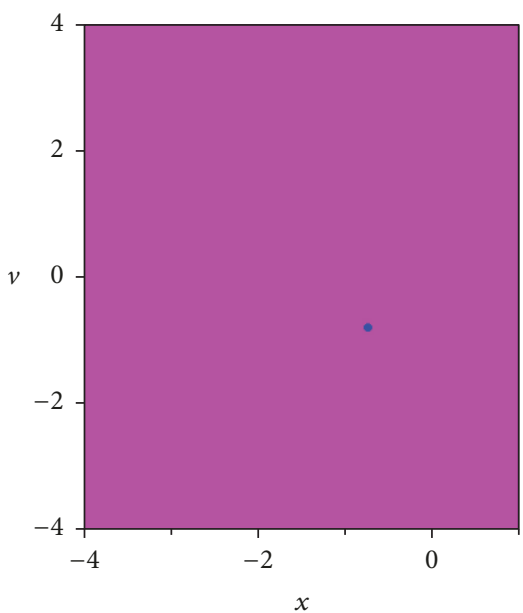

(f)

FIGURE 6: Evolution of the basins of attraction for the impact oscillator with bilinear damping obtained for $\omega=0.8, e=1, \xi_{1}=0.05, \xi_{2}=$ $0.04, r=0.7$, (a) $a=1.60$, (b) $a=1.65$, (c) $a=1.70$, (d) $a=1.95$, (e) $a=2.05$, and (f) $a=2.10$. Here the period-1 orbit with one impact per period shown in red has a cyan basin, and the period-1 orbit with two impacts per period shown in blue has a magenta basin.

\section{Appendix}

\section{The Analytical Solution of the Equation in} the Smooth Regimes $\Pi_{1}$ and $\Pi_{2}$

Consider the equation

$$
x^{\prime \prime}+2 \xi_{i} x^{\prime}+x=a \cos (\omega \tau), \quad i=1,2,
$$

with the initial conditions $x\left(\tau_{n}\right)=x_{n}, x^{\prime}\left(\tau_{n}\right)=v_{n}$. The solution is

$$
\begin{aligned}
x(\tau) & \\
= & A_{1} \cos (\omega \tau)+B_{1} \sin (\omega \tau)+e^{-\xi_{i}\left(\tau-\tau_{n}\right)} \\
& \times\left(C_{1} \sin \left(\gamma_{1}\left(\tau-\tau_{n}\right)\right)+C_{2} \cos \left(\gamma_{1}\left(\tau-\tau_{n}\right)\right)\right),
\end{aligned}
$$

and its directive is

$$
\begin{aligned}
& x^{\prime}(\tau)=-A \omega \sin (\omega \tau)+B_{1} \omega \cos (\omega \tau)+\left(-\xi_{i}\right) \\
& \cdot e^{-\xi_{i}\left(\tau-\tau_{n}\right)}\left(C_{1} \sin \left(\gamma_{1}\left(\tau-\tau_{n}\right)\right)\right. \\
& \left.+C_{2} \cos \left(\gamma_{1}\left(\tau-\tau_{n}\right)\right)\right) \\
& +e^{-\xi_{i}\left(\tau-\tau_{n}\right)}\left(C_{1} \gamma_{1} \cos \left(\gamma_{1}\left(\tau-\tau_{n}\right)\right)\right. \\
& \left.-C_{2} \gamma_{1} \sin \left(\gamma_{1}\left(\tau-\tau_{n}\right)\right)\right),
\end{aligned}
$$

where $A=a\left(1-\omega^{2}\right) /\left(\left(1-\omega^{2}\right)^{2}+(2 \xi \omega)^{2}\right), B_{1}=-2 a \xi_{i} \omega /((1-$ $\left.\left.\omega^{2}\right)^{2}+\left(2 \xi_{i} \omega\right)^{2}\right), \gamma_{1}=\sqrt{1-\left(\xi_{i}\right)^{2}}, C_{2}=x_{i}-\left(A_{1} \cos \left(\omega \tau_{n}\right)+\right.$ $\left.B_{1} \sin \left(\omega \tau_{n}\right)\right), C_{1}=\left(v_{n}+\xi_{i} C_{2}+A \omega \sin \left(\omega \tau_{n}\right)-B \omega \cos \left(\omega \tau_{n}\right)\right) / \gamma_{1}$.

\section{Disclosure}

This paper was presented at the International Conference on Engineering Vibration (Sofia, Bulgaria, September 4-7, 2017). 


\section{Conflicts of Interest}

The authors declare that there are no conflicts of interest regarding the publication of this paper.

\section{Acknowledgments}

This work is partially supported by the National Natural Science Foundation of China (Grants nos. 11402224, 11672257, and 11202180), the Natural Science Foundation of Jiangsu Province of China (Grants nos. BK20161314 and BK20151295), the Jiangsu Overseas Research and Training Program for University Prominent Young and Middle-Aged Teachers and Presidents, the Excellent Scientific and Technological Innovation Team of Jiangsu University, and Jiangsu Key Laboratory for Big Data of Psychology and Cognitive Science. The authors also thank Professor Marian Wiercigroch for helpful comments.

\section{References}

[1] S. W. Shaw and P. Holmes, "Periodically forced linear oscillator with impacts: chaos and long-period motions," Physical Review Letters, vol. 51, no. 8, pp. 623-626, 1983.

[2] G. S. Whiston, "The vibro-impact response of a harmonically excited and preloaded one-dimensional linear oscillator," Journal of Sound and Vibration, vol. 115, no. 2, pp. 303-319, 1987.

[3] A. B. Nordmark, "Non-periodic motion caused by grazing incidence in an impact oscillator," Journal of Sound and Vibration, vol. 145, no. 2, pp. 279-297, 1991.

[4] A. P. Ivanov, "Impact oscillations: linear theory of stability and bifurcations," Journal of Sound and Vibration, vol. 178, no. 3, pp. 361-378, 1994.

[5] S. Foale, "Analytical Determination of Bifurcations in an Impact Oscillator," Philosophical Transactions of the Royal Society A: Mathematical, Physical \& Engineering Sciences, vol. 347, no. 1683, pp. 353-364, 1994.

[6] S. Foale and S. R. Bishop, "Bifurcations in impact oscillations," Nonlinear Dynamics, vol. 6, no. 3, pp. 285-299, 1994.

[7] G. Luo and J. Xie, "Bifurcations and chaos in a system with impacts," Physica D: Nonlinear Phenomena, vol. 148, no. 3-4, pp. 183-200, 2001.

[8] A. C. J. Luo and L. Chen, "Periodic motions and grazing in a harmonically forced, piecewise, linear oscillator with impacts," Chaos, Solitons \& Fractals, vol. 24, no. 2, pp. 567-578, 2005.

[9] H. Dankowicz and X. Zhao, "Local analysis of co-dimensionone and co-dimension-two grazing bifurcations in impact microactuators," Physica D: Nonlinear Phenomena, vol. 202, no. 3-4, pp. 238-257, 2005.

[10] X. Zhao and H. Dankowicz, "Unfolding degenerate grazing dynamics in impact actuators," Nonlinearity, vol. 19, no. 2, pp. 399-418, 2006.

[11] P. Thota and H. Dankowicz, "Continuous and discontinuous grazing bifurcations in impacting oscillators," Physica D: Nonlinear Phenomena, vol. 214, no. 2, pp. 187-197, 2006.

[12] D. R. Chillingworth, "Discontinuity geometry for an impact oscillator," Dynamical Systems. An International Journal, vol. 17, no. 4, pp. 389-420, 2002.

[13] D. R. Chillingworth, "Dynamics of an impact oscillator near a degenerate graze," Nonlinearity, vol. 23, no. 11, pp. 2723-2748, 2010.
[14] N. Humphries and P. T. Piiroinen, "A discontinuity-geometry view of the relationship between saddle-node and grazing bifurcations," Physica D: Nonlinear Phenomena, vol. 241, no. 22, pp. 1911-1918, 2012.

[15] J. F. Mason and P. T. Piiroinen, "Saddle-point solutions and grazing bifurcations in an impacting system," Chaos: An Interdisciplinary Journal of Nonlinear Science, vol. 22, no. 1, 013106, 6 pages, 2012.

[16] H. Jiang, Y. Liu, L. Zhang, and J. Yu, "Anti-phase synchronization and symmetry-breaking bifurcation of impulsively coupled oscillators," Communications in Nonlinear Science and Numerical Simulation, vol. 39, pp. 199-208, 2016.

[17] H. Jiang, A. S. E. Chong, Y. Ueda, and M. Wiercigroch, "Grazing-induced bifurcations in impact oscillators with elastic and rigid constraints," International Journal of Mechanical Sciences, vol. 127, pp. 204-214, 2017.

[18] M. di Bernardo, C. J. Budd, and A. R. Champneys, PiecewiseSmooth Dynamical Systems: Theory and Applications, vol. 163 of Applied Mathematical Sciences, Springer, London, UK, 2008.

[19] Z. Szabó and A. Lukács, "Numerical stability analysis of a forced two-D.O.F. oscillator with bilinear damping," Journal of Computational and Nonlinear Dynamics, vol. 2, no. 3, pp. 211217, 2007.

[20] S. Natsiavas, "Stability of piecewise linear oscillators with viscous and dry friction damping," Journal of Sound and Vibration, vol. 217, no. 3, pp. 507-522, 1998.

[21] S. Natsiavas and G. Verros, "Dynamics of oscillators with strongly nonlinear asymmetric damping," Nonlinear Dynamics, vol. 20, no. 3, pp. 221-246, 1999.

[22] G. Verros, S. Natsiavas, and G. Stepan, "Control and dynamics of quarter-car models with dual-rate damping," Journal of Vibration and Control, vol. 6, no. 7, pp. 1045-1063, 2000.

[23] C. Rajalingham and S. Rakheja, "Influence of suspension damper asymmetry on vehicle vibration response to ground excitation," Journal of Sound and Vibration, vol. 266, no. 5, pp. 1117-1129, 2003.

[24] G. Verros, S. Natsiavas, and C. Papadimitriou, "Design optimization of quarter-car models with passive and semi-active suspensions under random road excitation," Journal of Vibration and Control, vol. 11, no. 5, pp. 581-606, 2005.

[25] Y. Liu, T. P. Waters, and M. J. Brennan, "A comparison of semi-active damping control strategies for vibration isolation of harmonic disturbances," Journal of Sound and Vibration, vol. 280, no. 1-2, pp. 21-39, 2005.

[26] C. Papalukopoulos and S. Natsiavas, "Nonlinear biodynamics of passengers coupled with quarter car models," Journal of Sound and Vibration, vol. 304, no. 1-2, pp. 50-71, 2007.

[27] A. C. Luo and A. Rajendran, "Periodic motions and stability in a semi-active suspension system with MR damping," Journal of Vibration and Control, vol. 13, no. 5, pp. 687-709, 2007.

[28] T. P. Waters, Y. Hyun, and M. J. Brennan, "The effect of dualrate suspension damping on vehicle response to transient road inputs," Journal of Vibration and Acoustics, vol. 131, no. 1, 8 pages, 2009.

[29] S. L. T. de Souza, I. L. Caldas, and R. L. Viana, "Damping control law for a chaotic impact oscillator," Chaos, Solitons \& Fractals, vol. 32, no. 2, pp. 745-750, 2007.

[30] Z. Sun and S. S. Ge, "Switched and impulsive systems: analysis, design and applications, Zhengguo Li, Yengchai Soh, Changyun Wen; Springer, Berlin. Heidelberg, 2005, ISBN: 3-540-23952-9.," Automatica, vol. 42, no. 9, pp. 1617-1620, 2006. 
[31] G. Xie and L. Wang, "Necessary and sufficient conditions for controllability and observability of switched impulsive control systems," Institute of Electrical and Electronics Engineers Transactions on Automatic Control, vol. 49, no. 6, pp. 960-966, 2004.

[32] Z.-H. Guan, D. J. Hill, and X. Shen, "On hybrid impulsive and switching systems and application to nonlinear control," Institute of Electrical and Electronics Engineers Transactions on Automatic Control, vol. 50, no. 7, pp. 1058-1062, 2005.

[33] G. Zong and Y. Wu, "Exponential stability of switched systems with impulsive effect," Control Theory and Technology, vol. 3, no. 1, pp. 60-66, 2005.

[34] Z. Zhang, "Robust Ho control of a class of discrete impulsive switched systems," Nonlinear Analysis, vol. 71, no. 12, pp. e2790e2796, 2009.

[35] H. Xu, X. Liu, and K. L. Teo, "Delay independent stability criteria of impulsive switched systems with time-invariant delays," Mathematical and Computer Modelling, vol. 47, no. 3-4, pp. 372-379, 2008.

[36] H. Xu, X. Liu, and K. L. Teo, "A LMI approach to stability analysis and synthesis of impulsive switched systems with time delays," Nonlinear Analysis: Hybrid Systems, vol. 2, no. 1, pp. 3850, 2008.

[37] X. Liu, S. Zhong, and X. Ding, "Robust exponential stability of nonlinear impulsive switched systems with time-varying delays," Lithuanian Association of Nonlinear Analysts. Nonlinear Analysis: Modelling and Control, vol. 17, no. 2, pp. 210-222, 2012.

[38] L. P. Zhang, H. B. Jiang, and Y. Liu, "Bifurcation analysis of a rigid impact oscillator with bilinear damping," in Proceedings of the Abstract, the International Conference on Engineering Vibration, Sofia, Bulgaria, 2017, http://www.icoev2017.org/ocs/ index.php/icoev/ICOEV2017/paper/view/242.

[39] J. Ing, E. Pavlovskaia, M. Wiercigroch, and S. Banerjee, "Experimental study of impact oscillator with one-sided elastic constraint," Philosophical Transactions of the Royal Society A: Mathematical, Physical \& Engineering Sciences, vol. 366, no. 1866, pp. 679-704, 2008.

[40] P. Thota and H. Dankowicz, "TC-HAT (TC): A novel toolbox for the continuation of periodic trajectories in hybrid dynamical systems," SIAM Journal on Applied Dynamical Systems, vol. 7, no. 4, pp. 1283-1322, 2008.

[41] H. Dankowicz and F. Schilder, "An extended continuation problem for bifurcation analysis in the presence of constraints," Journal of Computational and Nonlinear Dynamics, vol. 6, no. 3, Article ID 031003, 2011. 


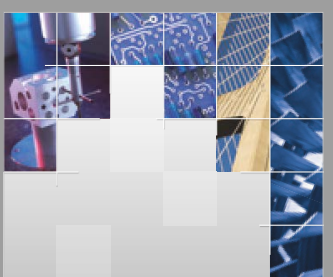

\section{Enfincering}
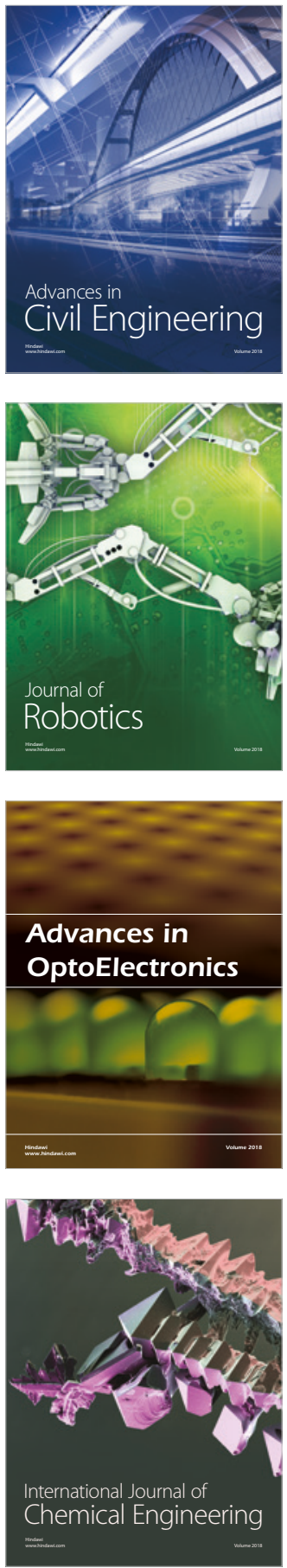

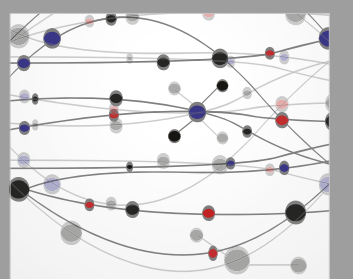

\section{Rotating \\ Machinery}

The Scientific World Journal

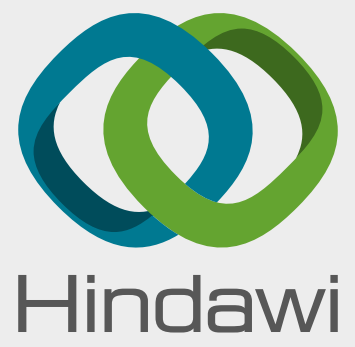

Submit your manuscripts at

www.hindawi.com
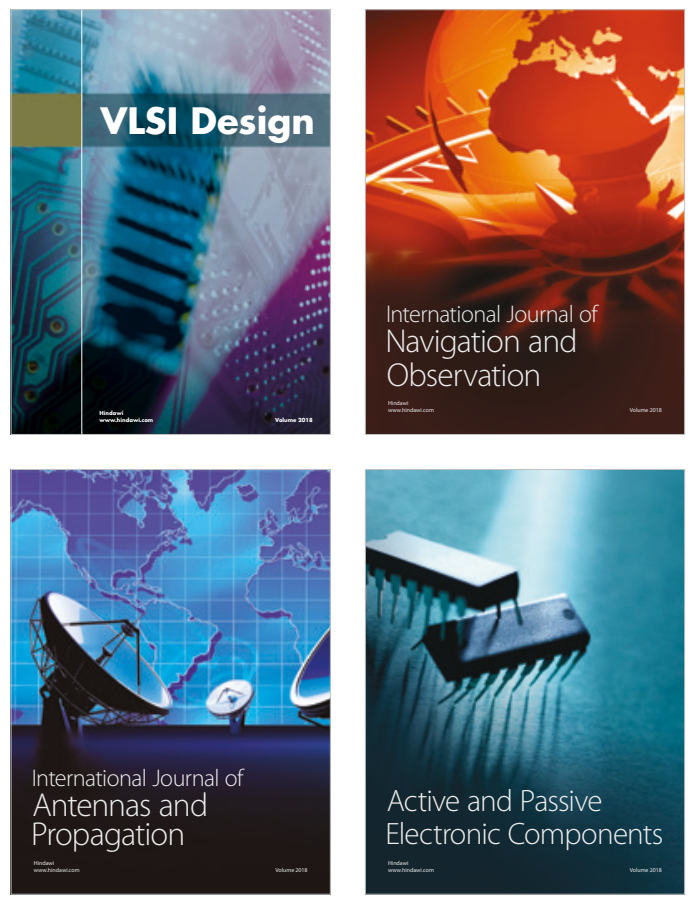
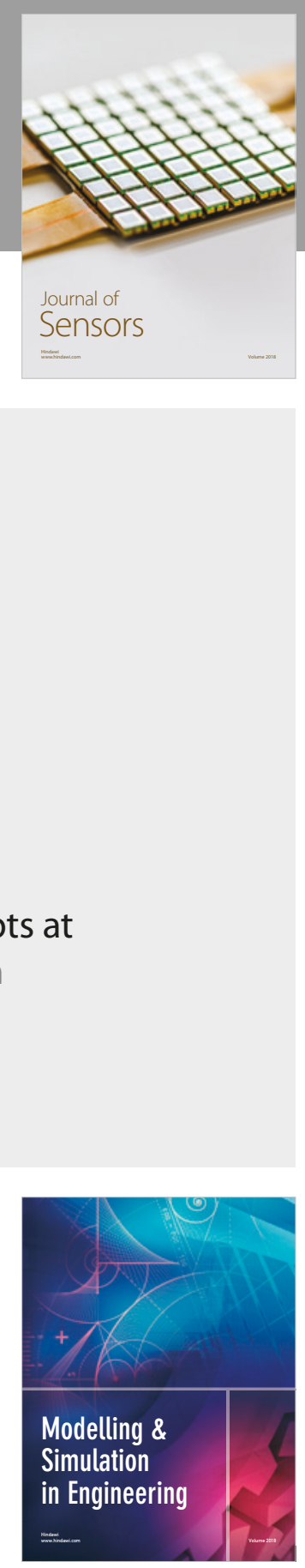

\section{Advances \\ Multimedia}
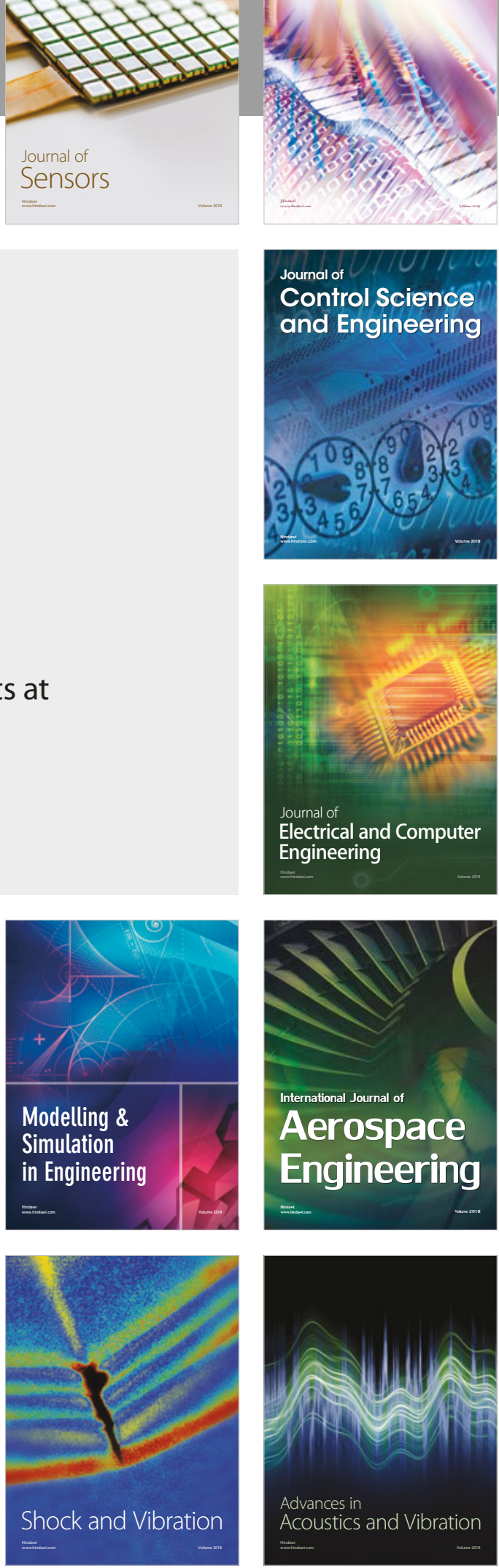